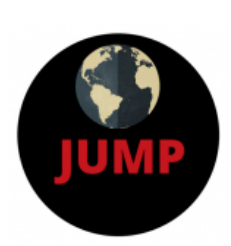

OJED

ISSN: 2574-3465 Print/ ISSN: 2574-3481 Online

Volume 3, Issue 2 (2019), pp. 99-108

(C) Journal of Underrepresented and Minority Progress

http://ojed.org/jump

\title{
The Relationship Between the Repeated Social Defeat Stress Experimental Model, Delegitimization, and Neuroresilience in Experiences of Young African- American Males
}

\author{
Regina Shepherd \\ Lehman College, USA
}

\section{ABSTRACT}

Repeated Social Defeat Stress is a mouse model in which depressive phenotyping is elicited by the application of stressful procedures and stressful events. Delegitimization is the process of egregiously and negatively stereotyping one group with the intention of excluding and harming a particularly targeted group by depriving it of visibility, legitimacy, and resources through dehumanization and marginalization. Based on a survey on delegitimization, the characterization and the experiences of delegitimized groups, it can be said that through the RSDS model we can understand the psychology and the real-life experiences of those in delegitimized groups like young African American males.

Keywords: Anxiety, Depression, Resilience, Stress

\section{INTRODUCTION}

This commentary highlights the relationship between the Repeated Social Defeat Stress (RSDS) experimental mouse model, the 
sociopsychological phenomenon known as 'delegitimization' and neuroresilience in the young Black males' experience of racism in America. The research on neuroresilience using the RSDS model constitutes groundbreaking work elucidating stress-induced depressive and anxiogenic symptomology in the hopes of detecting mechanisms that influence the functioning of the neuroimmune system. Highlighting this relationship allows at-risk populations like the young Black American male demographic to be propelled to the forefront of medical consciousness from the periphery, a vantage point bringing benefits drawn from the level of molecular to that of behavioral phenomenon as more research is done on stress-induced depression and anxiety.

Depression is the result of a collection of psychosocial stressors that have both biochemical and socioeconomic antecedents ${ }^{1}$. Racism is a stressful experience in America that systematically and categorically puts African Americans at risk for developing physiopsychological illnesses. Thus, racism attacks the ego of its victims through both blatant and subtle insults, as well as through negative imagery of Black people in the popular culture - the internalization of which can create anxieties and reactions that can lead to adverse psychological functioning. Studies have shown racism to be a public health issue $e^{3}$ linking it to the occurrence or onset of depression and even to exorbitant mortality rates among young Black American males. Everyday discrimination, chronic discrimination is significantly related to mental health status and is independent of other factors such as stressful life events and financial stress, for the perception of unfair treatment can induce psychological distress. Certain beliefs about Black inferiority have trickled down into policies that affect African Americans and restrict their educational and employment opportunities with the most obvious effects being found in racial disparities in residential opportunities and in socioeconomic status ${ }^{2}$.

Black people have a higher prevalence of psychological distress than do white people. Young Black males are subject to many socioeconomic factors that become stressors and affect their biochemistry negatively creating poor mental health outcomes for this demographic. Black men also are less able to identify that they have a mental health problem although they are typically overrepresented in mental health services ${ }^{5}$. Among the factors accounting for depression in young Black males are socioeconomic status, racial and masculine identity, self-esteem and mastery and quality healthcare ${ }^{6}$. Black men in the United States suffer disproportionately from illnesses and are victims of high mortality rates. When compared with other ethnic groups, Black men experience higher mortality rates in every leading cause of death, die about seven years earlier than do other groups, have higher 
rates of unemployment, experience higher rates of poverty, and are more likely to be exposed to racism, discrimination and violence and other situations that adversely affect mental health. Younger Black males are also more at risk for adverse health outcomes than are their more aged counterparts, as age is negatively correlated with depressive symptoms; thus older Black men have fewer depressive symptoms and lower levels of psychological distress ${ }^{7}$.

Factors like poverty level, education, employment and marital status are all significantly associated with depression. Those who have less than twelve years of formal education, tend to be more often unemployed and living at low poverty levels and, thus are more susceptible to having depressive symptoms. Young Black males are particularly more vulnerable because their experiences of psychic strain may directly influence the experiences they face throughout their middle adulthood ${ }^{8}$. It has been proven that stress is covariate with an abundance of diseases and social factors. Evaluating the young Black males' perception of stress is important as studies have indicated that there are significant relationships among race, degrees of skin color, sociodemographic statuses, blood pressure, or other measures of health.

\section{DISCUSSION}

A focus on the young African American male experience in America and their experiences with stress through racism is largely absent in the current research narratives of incidences of depression in the United States. There is a staggering lack in the literature about stress-induced depression precipitated by racism against young Black males.

The operative sociopsychological definition of stress is: the outcome of an individual's interactions with social institutions whose definitive element is to cause and sustain patterns of conflict, confusion and distress ${ }^{9}$. That racism can exacerbate the symptomology of depression and anxiety induced by stress is contained in the sociopsychological phenomena known as resilience and delegitimization. To be resilient in this context would mean that young Black males could be knowledgeable about the possibility of their belonging to a delegitimized entity, with of the limitations that come with it but behave in expressive ways that would be more useful than detrimental to them. Daniel Bar-Tal gives a definition of the sociopsychological phenomenon known as delegitimization and offers a synthesis of what it means to be a part of a delegitimized group. 
According to Bar-Tal, there are five main stages of delegitimization: dehumanization, trait characterization, outcasting, the use of political labels and group comparison ${ }^{10}$.

1. Dehumanization happens when groups of people are negatively categorized.

2. Delegitimization is a category of prejudice where a group can be said to possess traits that are extremely undesirable or unacceptable in a society ${ }^{10}$.

3. 'Outcasting' occurs when members of a delegitimized group are viewed as violators of major social norms - a violation which usually is so egregious that it leads to the exclusion from society or institutionalization.

4. Tensions are usually very high between groups when there is a use of political labels, because typically one group is transformed into a political entity that is viewed as threatening to other societal systems.

5. Cultural comparison is a hybrid of 'outcasting' and labeling where a group is assigned a negative labe $1^{10}$. Historically, Black men have been construed as hypersexual, lazy, and even violent ${ }^{11}$. They have been essentially demonized and have experienced being delegitimized.

Delegitimization can serve the tendency for ethnocentricism ${ }^{10}$. Understanding delegitimization assists us in understanding the amount of cognitive stress that the at-risk population of young African American male face on a daily basis.

Understanding the relationship among neuroresiliency, social defeat, and delegitimized groups means that there is an understanding that prominent features, indicators of phenotype such as skin color, hair type or color, and physiognomy, are more readily the bases for delegitimization when it occurs $^{10}$.

This history of the United States offers a prime example of how delegitimization is bred and cultivated. Slaves in this country were subjected to chronic social defeat and delegitimization, thus providing a justification for slavery. The language, religion, physical conditions and the humanity of Black people were derided; this was the main social order until African Americans arose and protested, demanding civil rights after the 1950s and 1960s. The trickle-down effects of slavery, however, carry on; even today, there still exists institutionalized racism and prejudice.

This becomes important for neuroresiliency because it is precisely under these conditions that we see the stressors and stressful situations 
multiply and, as they proliferate, medicine apparently is becoming less and less equipped to handle the ripple effects. Could it be that medicine is becoming just another institution that shirks its responsibility for the young Black male in America? Could it be that the psychological distress young Black males experience in America can be blown out of proportion? Perhaps a glimpse into the RSDS experimental model, which is used to elicit depressive and anxiogenic phenotypes in neuroresiliency research, can be a source that provides some delineation of the experience of the invisible battle these young men fight with recurrent, unavoidable institutionalized defeat.

The Repeated Social Defeat Stress Model is a way to explore those molecular events that underlie the behavioral outcomes and phenotypes of stress-induced depression and anxiety. It is an experimental design through which we can observe the experience of the young African American male in America. The process has three main stages: the selection of the CD-1 aggressive mouse, the social confrontations between the CD-1 aggressive mouse and the C57BL/6J smaller mouse, and subsequent confirmatory social avoidance of the smaller, subordinate $\mathrm{C} 57 \mathrm{BL} / 6 \mathrm{~J}$ mouse, which indicates its defeat. From these labs, neuroresiliency has indicated that our neuroimmune system effectively copes with multiple stressors and adapts to pathologies, while retaining the ability to return to optimal functioning, while permanently changed after insults.

The RSDS model helps to provide insight into resiliency and susceptibility, namely how individuals who are said to be resilient actively adapt to stressor conditions while pathological responses are found among those who are said to be susceptible. Current research shows that grapederived polyphenols may be able to attenuate depressive symptomology elicited from RSDS conditioning and heralds the possibility of regulating depressive and anxiogenic phenotypes through epigenetic modulation ${ }^{12}$.

The implications of this study are huge. It adds to our understanding of neuropsychiatric disorders by explicating the molecular mechanisms underlying neuroinflammation in the brain, namely the epigenetic modulation of phytochemicals dihydrocaffeic acid (DHCA) and malvidin-3'-O-glucoside (Mal-gluc $)^{12}$. This adds to an increasing amount of evidence that shows that there are several protective factors for stress-induced diseases like depression and anxiety, which means that there are ways that victims of discrimination can become proactive about preventing racism and microaggressions from ruining their health.

Research goes further to identify neuroinflammation as the culprit for stress-mediated disease pathogenesis ${ }^{13}, 14$. The research on the molecular cascade that spawns sickness behavior implicates cognitive decline and 
impairment as risk factors and the byproducts of a stress-induced neuroinflammatory response. Studies also support the conclusion that physiological dysregulation is amplified by environmental stress as portrayed by the presentation of the depressive and anxiogenic phenotype elicited by the same RSDS preclinical behavior mode $1^{15}$.

Neuroresilience research thus reveals the potency of the interplay between stress diathesis and neuropsychiatric disorder prognosis. It is the definitive overlay between society, stress, environment and physiology: they all interrelate to influence disorder development. What is known about depressive and anxiogenic symptomology from RSDS preclinical research can inform further research through clinical trials on the interaction social defeat stress from racism or discrimination has with the human neuroimmune system.

The repeated social defeat stress model reveals a condition that effectively resembles the affected state of the neuroimmune system when stressors in life attack the body. The social defeat paradigm provides a perfect vantage point from which to survey the stress young Black males experience as members of a delegitimized entity. Young Black males often face prejudice and the effects of institutionalized racism, knowingly or unknowingly, and can become traumatized by the encounter. The measure of despair and anxiety used in the RSDS model is a social avoidance test, with a defeated mouse usually preferring to remain isolated. The cognitive appraisal that might be present in a human model is important because it is a crucial part of phenotypic manifestation: depression, sadness and other sickness behavior represents an awareness of a hopeless, unjust reality. Are the results based on the individual and his summation of the events that have transpired?

The behavior of the smaller C57BL/6J mouse mirrors the behavior observed among young Black males across America after traumatizing experiences with the social system. Exposure to institutionalized injustice and disrespect can cause very poor psychological symptoms similar to those witnessed at the aftermath of RSDS conditioning. Just like the defeated mouse, young Black males can spiral into self-defeating attitudes, maladaptive and self-destructive behaviors and loss of hope or diminished aspirations for the future 9 .

When individuals feel as if they have no control over their circumstances or environment, they are likely to become depressed and withdrawn, comparable to the phenotypes witnessed in the defeated mice. ShouldThat the 'defeated' young African American male tend to survey his circumstances as harmful, he may be subject to a cascade of unremittingly negative emotional reactions including withdrawal, depression and shame ${ }^{3}$. 
There is a strong relationship between racial socialization, experienced discrimination or prejudice, and depression. Delegitimization magnifies that relationship. Proactive racial socialization is inversely related to depressive phenotyping 9 , which indicates that there is a connection between racial socialization and depressive phenotyping. Some become so fractured that they leave main society, join gangs, engage in self-defeating lifestyles while others turn to self-medicating or other means to numb the pain of living the life of a delegitimized entity.

The research on neuroresilience might promote a healthy neuroimmune system and functioning. Its future may be to reverse or prevent what has been ingrained through repeated defeat and the stress that comes from it. The work done in neuroresilience addresses the issue of stress-related psychological impairment on a molecular level for all who take seriously the gradual cognitive and social impairment caused by stress-induced experiences and diseases. It has been reported that at least $20 \%$ of patients with stress-related disorders also meet the criteria for having cognitive impairment ${ }^{13}$, making the issue of stress-related disorders a prominent one.

The conditions under which these maladjustments become prominent are equally important. There can be extrapolated the propensity for defeat and susceptibility by virtue of insurmountable stress not only in the RSDS experimental mouse model but also within the human realm. A similar behavioral paradigm is witnessed when analyzing the relationship between young African American males and American institutions. They have unique experiences because of their distinct contact with such institutions and they represent a peculiar segment of the population because of their experience with them. Thus, they endure a different kind of stress that must be understood to effectively anticipate any stress-related neuroimmune or cognitive deficiencies.

Delegitimization becomes important because given this country's historical context, we can see how a form of repeated social defeat led to submission to inhumane conditions. The stresses that arose from an adverse historical legacy gave birth, centuries later, to pockets of individuals within the African American population that have come to harvest social disregard. Systematic discrimination, historically, thus has increased the likelihood for susceptibility to illness of an entire group; repeated social defeat is endemic somewhere, in whatever form, in this historical experience.

\section{CONCLUSION}

The African American male experience of delegitimization in our culture has generated crippling effects well into the $21^{\text {st }}$ century. Young Black 
males have suffered from the lack of self-confidence and sadness that they have been compelled to feel when they are at the mercy of a world that they must care about but which does not care about them ${ }^{8}$. Racism catalyzes a gamete of stressful circumstances and current health care providers have proven unable to develop culturally aware and informative assessments, and appropriate interventions because there has been a lack in the systematic research that can construct an accurate psychological profile of delegitimized groups such as the young Black male in America ${ }^{3,1,5}$.

Stresses coming from repeated social defeat, delegitimization, and neuroresiliency trigger an unfortunate cascade of malfunctioning molecular mechanisms and phenotyping. This occurs when young Black American males experience repeated social defeat while facing institutionalized racism and every-day racism and discrimination. What we learn from the paradigm is that the stress literally builds or breaks a person. If a person overcomes such stresses, then his or her neuroimmune system adapts and the behavior follows a 'resilient' profile. He or she develops a neuroimmune system that is distinctly resilient in the face of the chronic stress. If a person is broken by such stress, however, then their neuroimmune system malfunctions and the behavior follows a 'susceptible' profile.

The study of neuroresilience focuses on the neuroimmune pathology of susceptibility to combat and it may help physicians to reverse the malfunction. Understanding neuroresilience at the molecular level and its relationship to stress-induced depression or other psychological pathologies can provide a framework from which to view the experience of stress the young African American male faces continually.

African American males represent a segment of the population that has been not only delegitimized by society but is also at risk for cognitive impairment as a result of experiencing repeated social defeat stress through their encounters with institutionalized social delegitimization. The parallel between this experimental model and the experience of racism by the young Black male not only makes the endured stress prominent, but it also points to the experience as possibly etiological to depressive and anxiogenic symptomology in this demographic.

The paradigm can be used both to elicit phenotype and to understand situational determinants of neuropsychiatric disease prognosis, and it offers a possible starting point for further research towards this effect. As more is learned about the basis of neurobiological resiliency, we can better understand psychosocial resiliency. Stress in the African American community is a force to be reckoned with, and its effects seem to be most pronounced among young Black males. African Americans are disproportionately diagnosed with stress- 
related diseases like hypertension, cardiovascular disease, stroke and substance/alcohol abuse. This is further complicated by other extenuating circumstances connected to sociodemographic variables, race, genetics, and the residual effects of the individual experience of institutionalized discrimination ${ }^{3}$.

The young Black male is constantly threatened by the debilitating effects of delegitimization. A young Black male's experience in this country cannot be assessed without the concession that racism is at some points an institutionalized construct and is thereby unavoidable. This group is subject to continuing discriminatory experiences as a socially delegitimized group. Thus, understanding the experiences of discrimination, racism, and delegitimization is central to assessing and addressing the health risks of this population and in turn its stress-induced disease prognosis. It can also lead to further research on perceived psychological insults, like racism and discrimination, on the neuroimmune system. This underserved and underrepresented group requires further medical science research to mitigate the devastating effects that malevolent society has created.

\section{REFERENCES}

Allen, N. L., Becerra, B. J., \& Becerra, M. B. (2018). Associations between food insecurity and the severity of psychological distress among AfricanAmericans. Ethnicity \& health, 23(5), 511-520.

Bar-Tal, D. (1990). Causes and consequences of delegitimization: Models of conflict and ethnocentrism. Journal of Social issues, 46(1), 65-81.

Blaze, Jennifer, et al. "Polyphenolic Compounds Alter Stress-Induced Patterns of Global DNA Methylation in Brain and Blood." Molecular nutrition \& food research 62.8 (2018): 1700722.

Keating, F. (2009). African and Caribbean men and mental health. Ethnicity and inequalities in Health and Social Care, 2(2), 41-53.

Lincoln, K. D., Taylor, R. J., Watkins, D. C., \& Chatters, L. M. (2011). Correlates of psychological distress and major depressive disorder among African American men. Research on Social Work Practice, 21(3), 278-288.

Outlaw, F. H. (1993). Stress and coping: The influence of racism of the cognitive appraisal processing of African Americans. Issues in Mental Health Nursing, 14(4), 399-409.http://dx.doi.org/10.3109/01612849309006902

Payne, Y. A. (2011). Site of resilience: A reconceptualization of resiliency and resilience in street life-oriented Black men. Journal of Black Psychology, 37(4), 426-451.

Pfau, M. L., \& Russo, S. J. (2016). Neuroinflammation regulates cognitive impairment in socially defeated mice. Trends in neurosciences, 39(6), 353355 . 
Radtke, F. A., Chapman, G., Hall, J., \& Syed, Y. A. (2017). Modulating

Neuroinflammation to treat neuropsychiatric disorders. BioMed research international, 2017.

Stevenson, H. C., Reed, J., Bodison, P., \& Bishop, A. (1997). Racism Stress

Management: Racial Socialization Beliefs and the Experience of Depression and Anger in African American Youth. Youth \& Society, 29(2), 197-222. https://doi.org/10.1177/0044118X97029002003

Watkins, D. C., Green, B. L., Rivers, B. M., \& Rowell, K. L. (2006). Depression and black men: Implications for future research. The journal of men's health \& gender, 3(3), 227-235.

Watkins D. C. (2011). Depression over the adult life course for African American men: toward a framework for research and practice. American journal of men's health, 6(3), 194-210.

Watkins, D. C., Hudson, D. L., Howard Caldwell, C., Siefert, K., \& Jackson, J. S. (2011). Discrimination, mastery, and depressive symptoms among African American men. Research on Social Work Practice, 21(3), 269-277.

Wang, Jun, et al. "Epigenetic modulation of inflammation and synaptic plasticity promotes resilience against stress in mice." Nature communications 9.1 (2018): 477.

Williams, D., \& Williams-Morris, R. (2000). Racism and mental health: The African American experience. Ethnicity and health, 5(3-4), 243-268.

REGINA SHEPHERD Email: regina.g.shepherd@gmail.com 